\title{
Successful catheter ablation above aortic sinus cusp approach eliminating the ventricular arrhythmia arising from the myocardial crescent beneath the interleaflet triangle : LGE-MRI assessment
}

\author{
kunihiko kiuchi ${ }^{1}$, Yu Izawa ${ }^{2}$, Hiroyuki Toh $^{2}$, and Ken-ichi Hirata ${ }^{1}$ \\ ${ }^{1}$ Kobe University Graduate School of Medicine \\ ${ }^{2}$ Kobe University Graduate School of Medicine School of Medicine
}

January 26, 2021

\begin{abstract}
A 61-year-old female with 50000 ventricular premature contractions and reduced left ventricular ejection fraction of $35 \%$ referred to our center. Although the origin was considered to be originated from the junction between the left and right coronary cusp, a single radiofrequency application above the aortic sinus cusp could eliminate it.
\end{abstract}

Successful catheter ablation above aortic sinus cusp approach eliminating the ventricular arrhythmia arising from the myocardial crescent beneath the interleaflet triangle : LGE-MRI assessment

Kunihiko Kiuchi, MD, Yu Izawa, MD, Hiroyuki Toh, MD, Ken-ichi Hirata, MD

Section of Arrhythmia, Division of Cardiovascular Medicine, Department of Internal Medicine, Kobe University Graduate School of Medicine

7-5-2 Kusunoki-chou chuou-ku, Kobe, Hyogo, Japan

Key Words: myocardial crescent; interleaflet triangle; ventricular arrhythmia; radiofrequency catheter ablation;

Conflict of interest

The Section of Arrhythmia is supported by an endowment from Medtronic JAPAN and Abbott JAPAN. The authors have reported that they have no relationship relevant to the contents of this paper to disclose.

Funding

None

Short title: Ventricular arrhythmia arising from myocardial crescent

Total word count: 1278

Address: Kunihiko Kiuchi, M.D, (corresponding author)

Section of Arrhythmia, Division of Cardiovascular Medicine, Department of Internal Medicine, Kobe University Graduate School of Medicine

7-5-2 Kusunoki-chou chuou-ku, Kobe, Hyogo, Japan

Fax: (81)-78-382-5859 
Telephone: (81)-78-382-5111

E-mail: kunihikokiuchi@yahoo.co.jp

\title{
Key Clinical Message
}

Late gadolinium enhancement magnetic resonance imaging (LGE-MRI) can visualize the radiofrequency $(\mathrm{RF})$ ablation lesion as well as pre-existing myocardial fibrosis. The late gadolinium enhancement area after $\mathrm{RF}$ application has a potential accurately to detect the origin of ventricular arrhythmia.

\begin{abstract}
A 61-year-old female with 50000 ventricular premature contractions and reduced left ventricular ejection fraction of $35 \%$ referred to our center. Although the origin was considered to be originated from the junction between the left and right coronary cusp, a single radiofrequency application above the aortic sinus cusp could eliminate it.
\end{abstract}

\section{Case history}

A 61-year-old female who slightly felt the dyspnea on effort and was admitted to our center. 24-hours electrocardiogram (ECG) showed a total of 50000 ventricular premature contractions (VPC) and the left ventricular ejection fraction (LVEF) was reduced by $35 \%$. Although the origin was likely originated from the junction between the left and right coronary cusp, catheter manipulation below the cusp was difficult due to the anatomical reason. Fortunately, a single radiofrequency application above the right coronary cusp (RCC) near the left coronary cusp (LCC) - right coronary cusp (RCC) junction could eliminate the VPCs. Her symptom disappeared immediately after the procedure, and no VPC recurred and LVEF was normalized 6 months after the procedure. LGE-MRI could excellently visualize the RF lesion at the myocardial crescent beneath the interleaflet triangle.

\section{Discussion}

Idiopathic ventricular arrhythmia (VA) from the ostium of the left ventricle could be eliminated by the radiofrequency (RF) application within the aortic sinus cusps (ASCs). The VA originating from the junction between the left and right coronary cusp was rare, however, the elimination needs to the RF application below the aortic cusps.[1] Of interest, this VA had a unique electrocardiographic characteristic of a qrS pattern in at least one of the leads V1-V3 or abrupt V3 transition (Figure 1A).[2, 3] The tip of the ablation catheter should be positioned at this site by deflecting the loop of the ablation catheter in the left ventricle cavity or on the non-coronary cusp, but this technique was relatively difficult to stabilize the ablation catheter due to the limited anatomical space. In the current case, although electrogram at the ablation catheter was sub-optimal, RF application at the left coronary cusp (LCC) - right coronary cusp (RCC) junction above ACSs could eliminate the VA (Figure 1C). The local electrogram at the successful ablation site showed a tiny dull ventricular potential with low $\mathrm{R}$ wave amplitude. The distal tip of the ablation catheter in the fluoroscopy was located behind the coronary sinus catheter in the right anterior oblique view (Figure 1B, C). These findings suggest the ablation catheter is more likely to be located close to the LCC-RCC junction above the ASCs.

To identify radiofrequency lesion extension, high-resolution late-gadolinium enhancement magnetic resonance imaging (LGE-MRI) was performed three months after the catheter ablation. The LGE-MRI of ASC and left ventricle was acquired using a 3D inversion recovery, respiration navigated, electrocardiogram-gated, T1-FFE sequence in the transverse plane 15 minutes after the contrast injection, as previously reported. The typical parameters were as follows: repetition time/echo time $=4.7 / 1.5 \mathrm{~ms}$, voxel size $=1.43 \times 1.43$ $\times 2.40 \mathrm{~mm}$ (reconstructed to $0.63 \times 0.63 \times 1.20 \mathrm{~mm}$ ), flip angle $=15^{\circ}, \mathrm{SENSE}=1.8$, and 80 reference lines. The inversion time (TI) was set at $280-320 \mathrm{~ms}$ using a Look-Locker scan. LGE-MRI could demonstrate that the strong LGE could be found at the ventricular myocardium beyond the LCC - RCC junction, so-called myocardial crescents. However, we have clearly demonstrated that the LCC - RCC junction does not involve the myocardial crescents. [4] The resign corresponds to the myocardium beneath the interleaflet triangle, 
which is located within the left ventricle. Therefore, it is better reached from the below ASC approach with caution to the thin interleaflet triangle. In the current case, fortunately, RF lesion could extent from the myocardial crescent beneath the LCC - RCC junction to the myocardium beneath the interleaflet triangle (Figure 2). In case when the accurate mapping and catheter stability was difficult beneath the ASCs, careful mapping and RF application at the LCC - RCC junction above the ASCs might be acceptable option to eliminate the VA arising from the myocardium beneath the interleaflet triangle.

\section{Acknowledgement}

none

\section{Figure Legends}

\section{Figure 1}

A: Twelve-lead electrocardiograms during a ventricular arrhythmia and sinus rhythm. Note that the qrS pattern in V1 and abrupt R-wave transition in V3. B: Activation map of the ASC (upper panel). Local electrogram at the successful ablation site (lower panel). The tiny dull ventricular signal preceded the QRS onset by $28 \mathrm{~ms}$. C: A single RF application could eliminate the VA (upper panel). Ablation catheter position at the success site in LAO 55 @ and RAO $30 @$ view (lower panel).

$\mathrm{ABL}=$ ablation catheter, $\mathrm{LAO}=$ left anterior oblique, $\mathrm{RAO}=$ right anterior oblique

\section{Figure 2}

A: Three-dimensionally reconstructed LGE-MRI of the RV, LV and ASC. The lower panel shows LV ostium with and without ASC in the cranial view. The late gadolinium enhancement area (RF lesion) was colored by the light green. B: RF lesion on the LGE-MRI. The light green arrowhead indicates the RF lesion at the myocardial crescent just beneath ASC where the RF lesion likely extent to the myocardial just beneath the RCC-LCC junction.

$\mathrm{ASC}=$ aortic sinus cusp, LAO $=$ left anterior oblique, $\mathrm{LCC}=$ left coronary cusp, $\mathrm{NCC}=$ non coronary cusp, $\mathrm{RAO}=$ right anterior oblique, $\mathrm{RCC}=$ right coronary cusp

\section{Authorship list}

Kunihiko Kiuchi: I mainly described the manuscript.

Yu Izawa: He contributed MRI and 3D visualization.

Hiroyuki Toh: He contributed MRI and 3D visualization.

Koji Fukuzawa: He advised the electrophysiological assessment of VT ablation.

Mitsuru Takami: He checked the imaging quality in the manuscript.

Ken-ich Hirata: He checked the discussion section in the manuscript.

\section{References}

1. Yamada, T., H. T. McElderry, H. Doppalapudi, Y. Murakami, Y. Yoshida, N. Yoshida, et al. 2008. Idiopathic ventricular arrhythmias originating from the aortic root prevalence, electrocardiographic and electrophysiologic characteristics, and results of radiofrequency catheter ablation. J Am Coll Cardiol. 52:139147.

2. Yamada, T., N. Yoshida, Y. Murakami, T. Okada, M. Muto, T. Murohara, et al. 2008. Electrocardiographic characteristics of ventricular arrhythmias originating from the junction of the left and right coronary sinuses of Valsalva in the aorta: the activation pattern as a rationale for the electrocardiographic characteristics. Heart Rhythm. 5:184-192. 
3. Liao, H., W. Wei, K. S. Tanager, F. Miele, G. A. Upadhyay, A. D. Beaser, et al. 2020. Left ventricular summit arrhythmias with an abrupt $\mathrm{V}(3)$ transition: Anatomy of the aortic interleaflet triangle vantage point. Heart Rhythm.

4. Toh, H., S. Mori, J. T. Tretter, Y. Izawa, S. Shimoyama, M. Suzuki, et al. 2020. Living Anatomy of the Ventricular Myocardial Crescents Supporting the Coronary Aortic Sinuses. Semin Thorac Cardiovasc Surg. $32: 230-241$.

\section{Figure1}

A

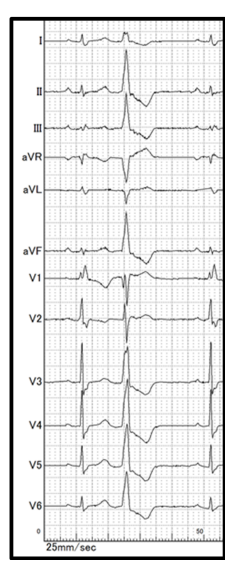

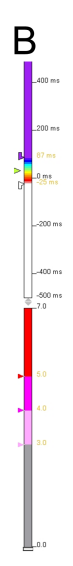
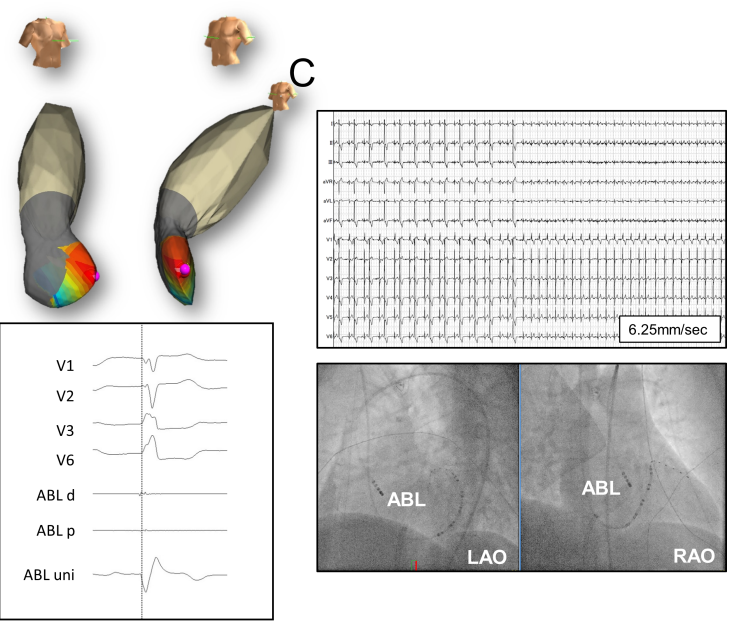

Figure2

A

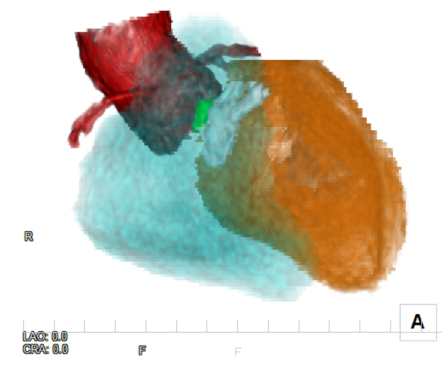

B
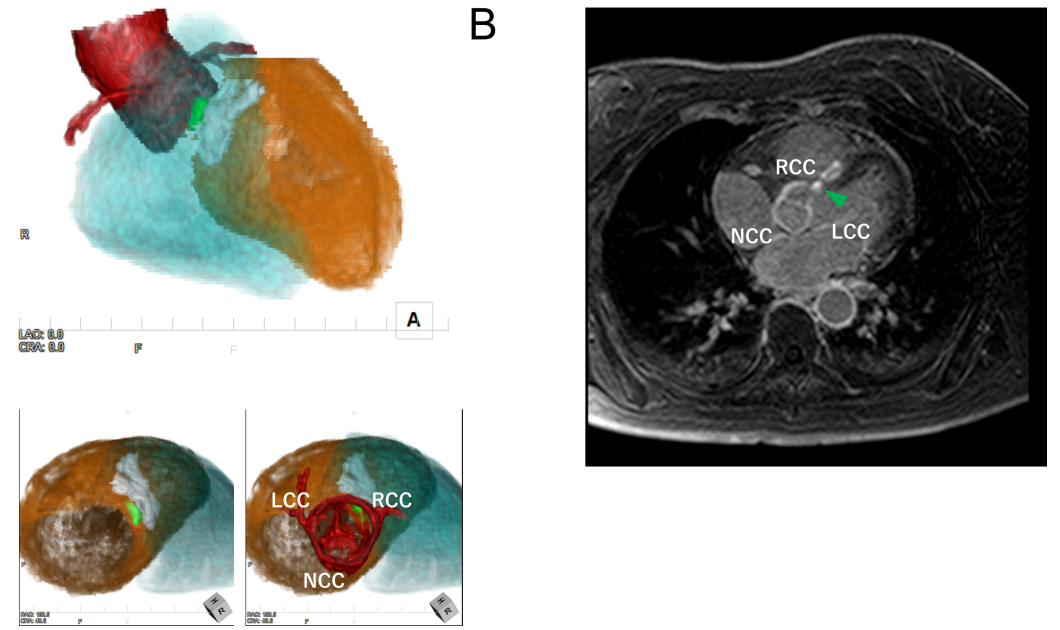